\title{
Classical soft graviton theorem rewritten
}

\author{
Biswajit Sahoo $^{a}$ and Ashoke Sen ${ }^{b}$ \\ ${ }^{a}$ Fields and Strings Laboratory, Institute of Physics, \\ Ecole Polytechnique Federale de Lausanne (EPFL), \\ CH-1015 Lausanne, Switzerland \\ ${ }^{b}$ Harish-Chandra Research Institute, HBNI, \\ Chhatnag Road, Jhusi, Allahabad 211019, India \\ E-mail: biswajit.sahoo@epfl.ch, sen@hri.res.in
}

ABSTRACT: Classical soft graviton theorem gives the gravitational wave-form at future null infinity at late retarded time $u$ for a general classical scattering. The large $u$ expansion has three known universal terms: the constant term, the term proportional to $1 / u$ and the term proportional to $\ln u / u^{2}$, whose coefficients are determined solely in terms of the momenta of incoming and the outgoing hard particles, including the momenta carried by outgoing gravitational and electromagnetic radiation produced during scattering. For the constant term, also known as the memory effect, the dependence on the momenta carried away by the final state radiation / massless particles is known as non-linear memory or null memory. It was shown earlier that for the coefficient of the $1 / u$ term the dependence on the momenta of the final state massless particles / radiation cancels and the result can be written solely in terms of the momenta of the incoming particles / radiation and the final state massive particles. In this note we show that the same result holds for the coefficient of the $\ln u / u^{2}$ term. Our result implies that for scattering of massless particles the coefficients of the $1 / u$ and $\ln u / u^{2}$ terms are determined solely by the incoming momenta, even if the particles coalesce to form a black hole and massless radiation. We use our result to compute the low frequency flux of gravitational radiation from the collision of massless particles at large impact parameter.

Keywords: Scattering Amplitudes, Classical Theories of Gravity, Effective Field Theories

ArXiv EPrint: 2105.08739 


\section{Contents}

1 Introduction and summary 1

2 Review of the analysis of the subleading term 4

3 Analysis of the subsubleading term $\quad 6$

4 Example involving scattering of massless particles $\quad 8$

5 Energy flux from massless particle scattering 12

6 Discussion 14

$\begin{array}{ll}\text { A Effect of electromagnetic interaction } & 15\end{array}$

\section{Introduction and summary}

Let us consider a general classical scattering process in which a set of $m$ objects carrying four momenta $p_{1}^{\prime}, \cdots, p_{m}^{\prime}$ in the asymptotic past come together, interact and then disperse as a set of $n$ objects carrying four momenta $p_{1}, \cdots p_{n}$. We shall choose the origin of our space-time coordinate system close to the region where the particles interact and consider a gravitational wave detector far away from the scattering region, whose space-time coordinates will be denoted by $(t, \vec{x})$. Our object of interest will be the gravitational wave-form at the detector:

$$
h_{\mu \nu}(t, \vec{x})=\frac{1}{2}\left(g_{\mu \nu}-\eta_{\mu \nu}\right),
$$

but we shall find it convenient to state the result in terms of a slightly different quantity that carries the same information:

$$
e_{\mu \nu} \equiv h_{\mu \nu}-\frac{1}{2} \eta_{\mu \nu} h_{\rho}^{\rho} \quad \Leftrightarrow \quad h_{\mu \nu} \equiv e_{\mu \nu}-\frac{1}{2} \eta_{\mu \nu} e_{\rho}^{\rho} .
$$

All indices are raised and lowered by the flat metric $\eta_{\rho \sigma}$. We also define

$$
R \equiv|\vec{x}|, \quad \hat{n}=\frac{\vec{x}}{R}, \quad n=(1, \hat{n}),
$$

and the retarded time at the detector:

$$
u=t-t_{0}, \quad t_{0}=\frac{R}{c}+\text { correction, }
$$

where $t_{0}$ is taken to be the time around which the peak of the gravitational wave-form reaches the detector. The 'correction' proportional to $\ln R$ represents the effect of the time delay due to gravitational drag on the gravitational waves due to the objects involved in the scattering. We denote by $G$ and $c$ respectively the Newton's gravitational constant and 
the speed of light in flat space-time. We also use mostly + signature metric and compute the inner products with flat metric unless mentioned otherwise. Since we are displaying explicit factors of $c$, the zeroth component of the momentum will be given by the energy divided by $c$. In this convention, results based on soft theorem determine the form of $e_{\mu \nu}$ at the detector, up to gauge transformation, at late and early retarded time [1-4]:

$$
\begin{aligned}
e_{\mu \nu} & =A_{\mu \nu}+\frac{1}{u} B_{\mu \nu}+u^{-2} \ln |u| F_{\mu \nu}+\mathcal{O}\left(u^{-2}\right)+\mathcal{O}\left(R^{-2}\right), & & \text { for large positive } u, \\
& =\frac{1}{u} C_{\mu \nu}+u^{-2} \ln |u| G_{\mu \nu}+\mathcal{O}\left(u^{-2}\right)+\mathcal{O}\left(R^{-2}\right), & & \text { for large negative } u,
\end{aligned}
$$

where,

$$
\begin{aligned}
& A^{\mu \nu}=\frac{2 G}{R c^{3}}\left[-\sum_{i=1}^{n} p_{i}^{\mu} p_{i}^{\nu} \frac{1}{n \cdot p_{i}}+\sum_{i=1}^{m} p_{i}^{\prime \mu} p_{i}^{\prime \nu} \frac{1}{n \cdot p_{i}^{\prime}}\right], \\
& B^{\mu \nu}=-\frac{4 G^{2}}{R c^{7}}\left[\sum_{i=1}^{n} \sum_{\substack{j=1 \\
j \neq i}}^{n} \frac{p_{i} \cdot p_{j}}{\left\{\left(p_{i} \cdot p_{j}\right)^{2}-p_{i}^{2} p_{j}^{2}\right\}^{3 / 2}}\left\{\frac{3}{2} p_{i}^{2} p_{j}^{2}-\left(p_{i} \cdot p_{j}\right)^{2}\right\} \frac{p_{i}^{\mu}}{n \cdot p_{i}}\left(n \cdot p_{j} p_{i}^{\nu}-n \cdot p_{i} p_{j}^{\nu}\right)\right. \\
& \left.-\left\{\sum_{j=1}^{n} p_{j} . n \sum_{i=1}^{n} \frac{1}{p_{i} \cdot n} p_{i}^{\mu} p_{i}^{\nu}-\sum_{j=1}^{m} p_{j}^{\prime} \cdot n \sum_{i=1}^{m} \frac{1}{p_{i}^{\prime} \cdot n} p_{i}^{\prime \mu} p_{i}^{\prime \nu}\right\}\right], \\
& C^{\mu \nu}=\frac{4 G^{2}}{R c^{7}}\left[\sum_{i=1}^{m} \sum_{\substack{j=1 \\
j \neq i}}^{m} \frac{p_{i}^{\prime} \cdot p_{j}^{\prime}}{\left\{\left(p_{i}^{\prime} \cdot p_{j}^{\prime}\right)^{2}-p_{i}^{\prime 2} p_{j}^{\prime 2}\right\}^{3 / 2}}\left\{\frac{3}{2} p_{i}^{\prime 2} p_{j}^{\prime 2}-\left(p_{i}^{\prime} \cdot p_{j}^{\prime}\right)^{2}\right\} \frac{p_{i}^{\prime \mu}}{n \cdot p_{i}^{\prime}}\left(n \cdot p_{j}^{\prime} p_{i}^{\prime \nu}-n \cdot p_{i}^{\prime} p_{j}^{\prime \nu}\right)\right], \\
& F^{\mu \nu}=\frac{2 G^{3}}{R c^{11}}\left[4 \sum_{j=1}^{n} p_{j} \cdot n \sum_{\ell=1}^{n} p_{\ell} \cdot n \sum_{i=1}^{n} \frac{p_{i}^{\mu} p_{i}^{\nu}}{p_{i} \cdot n}-4 \sum_{j=1}^{m} p_{j}^{\prime} \cdot n \sum_{\ell=1}^{m} p_{\ell}^{\prime} \cdot n \sum_{i=1}^{m} \frac{p_{i}^{\prime \mu} p_{i}^{\prime \nu}}{p_{i}^{\prime} \cdot n}\right. \\
& +4 \sum_{\ell=1}^{n} p_{\ell} \cdot n \sum_{i=1}^{n} \sum_{\substack{j=1 \\
j \neq i}}^{n} \frac{1}{p_{i} \cdot n} \\
& \times \frac{p_{i} \cdot p_{j}}{\left\{\left(p_{i} \cdot p_{j}\right)^{2}-p_{i}^{2} p_{j}^{2}\right\}^{3 / 2}}\left\{2\left(p_{i} \cdot p_{j}\right)^{2}-3 p_{i}^{2} p_{j}^{2}\right\}\left\{n \cdot p_{j} p_{i}^{\mu} p_{i}^{\nu}-n \cdot p_{i} p_{i}^{\mu} p_{j}^{\nu}\right\} \\
& +2 \sum_{\ell=1}^{m} p_{\ell}^{\prime} \cdot n \sum_{i=1}^{m} \sum_{\substack{j=1 \\
j \neq i}}^{m} \frac{1}{p_{i}^{\prime} \cdot n} \\
& \times \frac{p_{i}^{\prime} \cdot p_{j}^{\prime}}{\left\{\left(p_{i}^{\prime} \cdot p_{j}^{\prime}\right)^{2}-p_{i}^{\prime 2} p_{j}^{\prime 2}\right\}^{3 / 2}}\left\{2\left(p_{i}^{\prime} \cdot p_{j}^{\prime}\right)^{2}-3 p_{i}^{\prime 2} p_{j}^{\prime 2}\right\}\left\{n \cdot p_{j}^{\prime} p_{i}^{\prime \mu} p_{i}^{\prime \nu}-n \cdot p_{i}^{\prime} p_{i}^{\prime \mu} p_{j}^{\prime \nu}\right\} \\
& +\sum_{i=1}^{n} \sum_{\substack{j=1 \\
j \neq i}}^{n} \sum_{\substack{\ell=1 \\
\ell \neq i}}^{n} \frac{1}{p_{i} \cdot n} \frac{p_{i} \cdot p_{j}}{\left\{\left(p_{i} \cdot p_{j}\right)^{2}-p_{i}^{2} p_{j}^{2}\right\}^{3 / 2}}\left\{2\left(p_{i} \cdot p_{j}\right)^{2}-3 p_{i}^{2} p_{j}^{2}\right\} \\
& \left.\times \frac{p_{i} \cdot p_{\ell}}{\left\{\left(p_{i} \cdot p_{\ell}\right)^{2}-p_{i}^{2} p_{\ell}^{2}\right\}^{3 / 2}}\left\{2\left(p_{i} \cdot p_{\ell}\right)^{2}-3 p_{i}^{2} p_{\ell}^{2}\right\}\left\{n \cdot p_{j} p_{i}^{\mu}-n \cdot p_{i} p_{j}^{\mu}\right\}\left\{n \cdot p_{\ell} p_{i}^{\nu}-n \cdot p_{i} p_{\ell}^{\nu}\right\}\right],
\end{aligned}
$$

and

$$
\begin{aligned}
G^{\mu \nu}=-\frac{2 G^{3}}{R c^{11}}\left[2 \sum_{\ell=1}^{m} p_{\ell}^{\prime} \cdot n \sum_{i=1}^{m} \sum_{\substack{j=1 \\
j \neq i}}^{m} \frac{1}{p_{i}^{\prime} \cdot n}\right. \\
\quad \times \frac{p_{i}^{\prime} \cdot p_{j}^{\prime}}{\left\{\left(p_{i}^{\prime} \cdot p_{j}^{\prime}\right)^{2}-p_{i}^{\prime 2} p_{j}^{\prime 2}\right\}^{3 / 2}}\left\{2\left(p_{i}^{\prime} \cdot p_{j}^{\prime}\right)^{2}-3 p_{i}^{\prime 2} p_{j}^{\prime 2}\right\}\left\{n \cdot p_{j}^{\prime} p_{i}^{\prime \mu} p_{i}^{\prime \nu}-n \cdot p_{i}^{\prime} p_{i}^{\prime \mu} p_{j}^{\prime \nu}\right\}
\end{aligned}
$$




$$
\begin{aligned}
& -\sum_{i=1}^{m} \sum_{\substack{j=1 \\
j \neq i}}^{m} \sum_{\substack{\ell=1 \\
\ell \neq i}}^{m} \frac{1}{p_{i}^{\prime} \cdot n} \frac{p_{i}^{\prime} \cdot p_{j}^{\prime}}{\left\{\left(p_{i}^{\prime} \cdot p_{j}^{\prime}\right)^{2}-p_{i}^{\prime 2} p_{j}^{\prime 2}\right\}^{3 / 2}}\left\{2\left(p_{i}^{\prime} \cdot p_{j}^{\prime}\right)^{2}-3 p_{i}^{\prime 2} p_{j}^{\prime 2}\right\} \frac{p_{i}^{\prime} \cdot p_{\ell}^{\prime}}{\left\{\left(p_{i}^{\prime} \cdot p_{\ell}^{\prime}\right)^{2}-p_{i}^{\prime 2} p_{\ell}^{\prime 2}\right\}^{3 / 2}} \\
& \left.\quad \times\left\{2\left(p_{i}^{\prime} \cdot p_{\ell}^{\prime}\right)^{2}-3 p_{i}^{\prime 2} p_{\ell}^{\prime 2}\right\}\left\{n \cdot p_{j}^{\prime} p_{i}^{\prime \mu}-n \cdot p_{i}^{\prime} p_{j}^{\prime \mu}\right\}\left\{n \cdot p_{\ell}^{\prime} p_{i}^{\prime \nu}-n \cdot p_{i}^{\prime} p_{\ell}^{\prime \nu}\right\}\right]
\end{aligned}
$$

Note that the coefficients $A^{\mu \nu}, B^{\mu \nu}, C^{\mu \nu}, F^{\mu \nu}$ and $G^{\mu \nu}$ are given only by the momenta of the incoming and the outgoing objects and do not depend on the details of the scattering process. One can also check that each of these coefficients is gauge invariant, i.e. vanishes upon contraction with the four vector $n$.

In the above formulæ, $A_{\mu \nu}$ represents a permanent change in metric due to the passage of the gravitational wave, and is known as the memory effect [5-14]. Its connection to the leading soft graviton theorem has been discussed in [15]. The coefficients $B_{\mu \nu}$ and $C_{\mu \nu}$, representing long range tail of the gravitational wave-form, are related to the logarithmic correction to the subleading soft graviton theorem [3]. The coefficients $F_{\mu \nu}$ and $G_{\mu \nu}$ are related to the leading logarithmic correction to the subsubleading soft graviton theorem $[3,4]$.

If a significant fraction of energy is carried away by radiation, then the sum over the final state momenta in the expressions for $A_{\mu \nu}, B_{\mu \nu}$ and $F_{\mu \nu}$ should include integration over outgoing flux of radiation, regarded as a flux of massless particles. $C_{\mu \nu}$ and $G_{\mu \nu}$ are given in terms of incoming momenta only and are not sensitive to the momenta of outgoing particles or radiation.

The contribution to $A_{\mu \nu}$ due to the final state gravitational waves is some time referred to as non-linear memory [9] or null memory [14]. This makes the computation of $A_{\mu \nu}$ somewhat hard since we need to first find the angular distribution of the flux of energy carried away by the gravitational waves. A priori, computation of $B_{\mu \nu}$ and $F_{\mu \nu}$ suffers from the same difficulty. However it was found in [2] that due to some miraculous cancellations, the contribution to $B_{\mu \nu}$ due to final state massless particles, including gravitational waves, can be expressed in terms of the momenta of massive objects only. If we denote by $\tilde{p}_{i}$ the momenta carried by the final state massive objects only and by $\tilde{n}$ the number of such objects, then the modified formula takes the form:

$$
\begin{aligned}
B^{\mu \nu}= & -\frac{4 G^{2}}{R c^{7}}\left[\sum_{i=1}^{\tilde{n}} \sum_{\substack{j=1 \\
j \neq i}}^{\tilde{n}} \frac{\tilde{p}_{i} \cdot \tilde{p}_{j}}{\left\{\left(\tilde{p}_{i} \cdot \tilde{p}_{j}\right)^{2}-\tilde{p}_{i}^{2} \tilde{p}_{j}^{2}\right\}^{3 / 2}}\left\{\frac{3}{2} \tilde{p}_{i}^{2} \tilde{p}_{j}^{2}-\left(\tilde{p}_{i} \cdot \tilde{p}_{j}\right)^{2}\right\} \frac{\tilde{p}_{i}^{\mu}}{n \cdot \tilde{p}_{i}}\left(n \cdot \tilde{p}_{j} \tilde{p}_{i}^{\nu}-n \cdot \tilde{p}_{i} \tilde{p}_{j}^{\nu}\right)\right. \\
& \left.-\left\{\sum_{j=1}^{\tilde{n}} \tilde{p}_{j} \cdot n \sum_{i=1}^{\tilde{n}} \frac{1}{\tilde{p}_{i} \cdot n} \tilde{p}_{i}^{\mu} \tilde{p}_{i}^{\nu}-\sum_{j=1}^{m} p_{j}^{\prime} \cdot n \sum_{i=1}^{m} \frac{1}{p_{i}^{\prime} \cdot n} p_{i}^{\prime \mu} p_{i}^{\prime \nu}\right\}+\tilde{P}^{\mu} \tilde{P}^{\nu}-P^{\prime \mu} P^{\prime \nu}\right], \quad(1.10)
\end{aligned}
$$

where

$$
P^{\prime}=\sum_{i=1}^{m} p_{i}^{\prime}, \quad \tilde{P}=\sum_{i=1}^{\tilde{n}} \tilde{p}_{i} .
$$

We emphasize that (1.10) is not an independent formulæ but follows from (1.7) after setting $p_{i}^{2}=0$ for the massless final state particles. 
In this paper we shall show that a similar rewriting is possible for the quantity $F^{\mu \nu}$ as well. In particular, the expression for $F^{\mu \nu}$ can be manipulated into the form:

$$
\begin{aligned}
& F^{\mu \nu}=\frac{2 G^{3}}{R c^{11}}\left[4 \sum_{j=1}^{\tilde{n}} \tilde{p}_{j} \cdot n \sum_{\ell=1}^{\tilde{n}} \tilde{p}_{\ell} \cdot n \sum_{i=1}^{\tilde{n}} \frac{\tilde{p}_{i}^{\mu} \tilde{p}_{i}^{\nu}}{\tilde{p}_{i} \cdot n}-4 \sum_{j=1}^{m} p_{j}^{\prime} \cdot n \sum_{\ell=1}^{m} p_{\ell}^{\prime} \cdot n \sum_{i=1}^{m} \frac{p_{i}^{\prime \mu} p_{i}^{\prime \nu}}{p_{i}^{\prime} \cdot n}\right. \\
& +4 \sum_{\ell=1}^{\tilde{n}} \tilde{p}_{\ell} \cdot n \sum_{i=1}^{\tilde{n}} \sum_{\substack{j=1 \\
j \neq i}}^{\tilde{n}} \frac{1}{\tilde{p}_{i} \cdot n} \\
& \times \frac{\tilde{p}_{i} \cdot \tilde{p}_{j}}{\left\{\left(\tilde{p}_{i} \cdot \tilde{p}_{j}\right)^{2}-\tilde{p}_{i}^{2} \tilde{p}_{j}^{2}\right\}^{3 / 2}}\left\{2\left(\tilde{p}_{i} \cdot \tilde{p}_{j}\right)^{2}-3 \tilde{p}_{i}^{2} \tilde{p}_{j}^{2}\right\}\left\{n \cdot \tilde{p}_{j} \tilde{p}_{i}^{\mu} \tilde{p}_{i}^{\nu}-n \cdot \tilde{p}_{i} \tilde{p}_{i}^{\mu} \tilde{p}_{j}^{\nu}\right\} \\
& +2 \sum_{\ell=1}^{m} p_{\ell}^{\prime} \cdot n \sum_{i=1}^{m} \sum_{\substack{j=1 \\
j \neq i}}^{m} \frac{1}{p_{i}^{\prime} \cdot n} \\
& \times \frac{p_{i}^{\prime} \cdot p_{j}^{\prime}}{\left\{\left(p_{i}^{\prime} \cdot p_{j}^{\prime}\right)^{2}-p_{i}^{\prime 2} p_{j}^{\prime 2}\right\}^{3 / 2}}\left\{2\left(p_{i}^{\prime} \cdot p_{j}^{\prime}\right)^{2}-3 p_{i}^{\prime 2} p_{j}^{\prime 2}\right\}\left\{n \cdot p_{j}^{\prime} p_{i}^{\prime \mu} p_{i}^{\prime \nu}-n \cdot p_{i}^{\prime} p_{i}^{\prime \mu} p_{j}^{\prime \nu}\right\} \\
& +\sum_{i=1}^{\tilde{n}} \sum_{\substack{j=1 \\
j \neq i}}^{\tilde{n}} \sum_{\ell=1}^{\tilde{n}} \frac{1}{\tilde{p}_{i \neq i} \cdot n} \frac{\tilde{p}_{i} \cdot \tilde{p}_{j}}{\left\{\left(\tilde{p}_{i} \cdot \tilde{p}_{j}\right)^{2}-\tilde{p}_{i}^{2} \tilde{p}_{j}^{2}\right\}^{3 / 2}}\left\{2\left(\tilde{p}_{i} \cdot \tilde{p}_{j}\right)^{2}-3 \tilde{p}_{i}^{2} \tilde{p}_{j}^{2}\right\} \frac{\tilde{p}_{i} \cdot \tilde{p}_{\ell}}{\left\{\left(\tilde{p}_{i} \cdot \tilde{p}_{\ell}\right)^{2}-\tilde{p}_{i}^{2} \tilde{p}_{\ell}^{2}\right\}^{3 / 2}} \\
& \times\left\{2\left(\tilde{p}_{i} \cdot \tilde{p}_{\ell}\right)^{2}-3 \tilde{p}_{i}^{2} \tilde{p}_{\ell}^{2}\right\}\left\{n \cdot \tilde{p}_{j} \tilde{p}_{i}^{\mu}-n . \tilde{p}_{i} \tilde{p}_{j}^{\mu}\right\}\left\{n . \tilde{p}_{\ell} \tilde{p}_{i}^{\nu}-n . \tilde{p}_{i} \tilde{p}_{\ell}^{\nu}\right\} \\
& \left.+4 n . P^{\prime} P^{\prime \mu} P^{\prime \nu}-4 n . \tilde{P} \tilde{P}^{\mu} \tilde{P}^{\nu}\right] .
\end{aligned}
$$

This corresponds to restricting the sum over final state particles in (1.8) to over massive particles only, and adding the compensating term given in the last line of (1.12).

It follows from (1.10) and (1.12) that if the final state contains only massless particles, then $B_{\mu \nu}$ and $F_{\mu \nu}$ will depend on only the momenta of the objects in the initial state. In fact it can also be seen with little effort that if the final state contains one massive object and arbitrary number of massless objects, then $B_{\mu \nu}$ and $F_{\mu \nu}$ still depend only on the momenta of the objects in the initial state. In particular the terms proportional to $\tilde{P}^{\mu} \tilde{P}^{\nu}$ in these expressions exactly cancel the terms involving $\tilde{p}_{i}$.

Finally we would like to mention that if some of the initial and /or final state particles are charged then we also need to take into account the effect of long range electromagnetic interaction among these particles. These effects have been studied in [2-4]. Following the same procedure that will be described in section 2 and section 3 , it is easy to show that even in the presence of charged particles in the initial and the final states, the late time gravitational wave-form at future null infinity continues to be independent of the individual momenta of the final state massless particles. This has been demonstrated in appendix A.

In section 4 and 5 we apply our results to analyze gravitational radiation emitted during scattering of massless particles and compare the results with those in [16-18].

\section{Review of the analysis of the subleading term}

In this section we shall briefly review the analysis of the subleading term leading to (1.10). For this we divide the set of final momenta $\left\{p_{i}\right\}$ into the massive particle momenta $\tilde{p}_{i}$ and 
the massless particle momenta $\left\{\hat{p}_{i}\right\}$. We now use the fact that if either $p_{i}$ or $p_{j}$ represents a massless particle, then we have:

$$
\frac{p_{i} \cdot p_{j}}{\left\{\left(p_{i} \cdot p_{j}\right)^{2}-p_{i}^{2} p_{j}^{2}\right\}^{3 / 2}}\left\{\frac{3}{2} p_{i}^{2} p_{j}^{2}-\left(p_{i} \cdot p_{j}\right)^{2}\right\}=1 .
$$

Therefore the first term in the expression for $B^{\mu \nu}$ given in (1.7) may be written as:

$$
\begin{aligned}
\sum_{i=1}^{n} \sum_{\substack{j=1 \\
j \neq i}}^{n} \frac{p_{i} \cdot p_{j}}{\left\{\left(p_{i} \cdot p_{j}\right)^{2}-p_{i}^{2} p_{j}^{2}\right\}^{3 / 2}}\left\{\frac{3}{2} p_{i}^{2} p_{j}^{2}-\left(p_{i} \cdot p_{j}\right)^{2}\right\} \frac{p_{i}^{\mu}}{n \cdot p_{i}}\left(n \cdot p_{j} p_{i}^{\nu}-n \cdot p_{i} p_{j}^{\nu}\right) \\
=\sum_{i=1}^{\tilde{n}} \sum_{\substack{j=1 \\
j \neq i}}^{\tilde{n}} \frac{\tilde{p}_{i} \cdot \tilde{p}_{j}}{\left\{\left(\tilde{p}_{i} \cdot \tilde{p}_{j}\right)^{2}-\tilde{p}_{i}^{2} \tilde{p}_{j}^{2}\right\}^{3 / 2}}\left\{\frac{3}{2} \tilde{p}_{i}^{2} \tilde{p}_{j}^{2}-\left(\tilde{p}_{i} \cdot \tilde{p}_{j}\right)^{2}\right\} \frac{\tilde{p}_{i}^{\mu}}{n \cdot \tilde{p}_{i}}\left(n \cdot \tilde{p}_{j} \tilde{p}_{i}^{\nu}-n \cdot \tilde{p}_{i} \tilde{p}_{j}^{\nu}\right) \\
\quad+\sum_{i} \sum_{j} \frac{\tilde{p}_{i}^{\mu}}{n \cdot \tilde{p}_{i}}\left(n \cdot \hat{p}_{j} \tilde{p}_{i}^{\nu}-n \cdot \tilde{p}_{i} \hat{p}_{j}^{\nu}\right)+\sum_{i} \sum_{j} \frac{\hat{p}_{i}^{\mu}}{n \cdot \hat{p}_{i}}\left(n \cdot \tilde{p}_{j} \hat{p}_{i}^{\nu}-n \cdot \hat{p}_{i} \tilde{p}_{j}^{\nu}\right) \\
\quad+\sum_{i} \sum_{j} \frac{\hat{p}_{i}^{\mu}}{n \cdot \hat{p}_{i}}\left(n \cdot \hat{p}_{j} \hat{p}_{i}^{\nu}-n \cdot \hat{p}_{i} \hat{p}_{j}^{\nu}\right) .
\end{aligned}
$$

We can now use the result of momentum conservation:

$$
\sum_{j} \tilde{p}_{j}^{\nu}+\sum_{j} \hat{p}_{j}^{\nu}=P^{\prime \nu}, \quad P^{\prime} \equiv \sum_{i} p_{i}^{\prime}
$$

to express (2.2) as,

$$
\begin{aligned}
\sum_{i=1}^{n} \sum_{\substack{j=1 \\
j \neq i}}^{n} & \frac{p_{i} \cdot p_{j}}{\left\{\left(p_{i} \cdot p_{j}\right)^{2}-p_{i}^{2} p_{j}^{2}\right\}^{3 / 2}}\left\{\frac{3}{2} p_{i}^{2} p_{j}^{2}-\left(p_{i} \cdot p_{j}\right)^{2}\right\} \frac{p_{i}^{\mu}}{n \cdot p_{i}}\left(n \cdot p_{j} p_{i}^{\nu}-n \cdot p_{i} p_{j}^{\nu}\right) \\
= & \sum_{\substack { i=1 \\
\begin{subarray}{c}{n \\
j \neq i{ i = 1 \\
\begin{subarray} { c } { n \\
j \neq i } }\end{subarray}} \sum_{\substack{\tilde{n} \\
j \neq i}} \frac{\tilde{p}_{i} \cdot \tilde{p}_{j}}{\left\{\left(\tilde{p}_{i} \cdot \tilde{p}_{j}\right)^{2}-\tilde{p}_{i}^{2} \tilde{p}_{j}^{2}\right\}^{3 / 2}}\left\{\frac{3}{2} \tilde{p}_{i}^{2} \tilde{p}_{j}^{2}-\left(\tilde{p}_{i} \cdot \tilde{p}_{j}\right)^{2}\right\} \frac{\tilde{p}_{i}^{\mu}}{n \cdot \tilde{p}_{i}}\left(n \cdot \tilde{p}_{j} \tilde{p}_{i}^{\nu}-n \cdot \tilde{p}_{i} \tilde{p}_{j}^{\nu}\right) \\
& +\sum_{i} \frac{\tilde{p}_{i}^{\mu} \tilde{p}_{i}^{\nu}}{n \cdot \tilde{p}_{i}} n \cdot\left(P^{\prime}-\tilde{P}\right)+\sum_{i} \frac{\hat{p}_{i}^{\mu} \hat{p}_{i}^{\nu}}{n \cdot \hat{p}_{i}} n \cdot P^{\prime}+\tilde{P}^{\mu} \tilde{P}^{\nu}-P^{\prime \mu} P^{\prime \nu} .
\end{aligned}
$$

We also have

$$
-\sum_{j=1}^{n} p_{j} \cdot n \sum_{i=1}^{n} \frac{1}{p_{i} \cdot n} p_{i}^{\mu} p_{i}^{\nu}=-n \cdot P^{\prime} \sum_{i=1}^{n} \frac{1}{\tilde{p}_{i} \cdot n} \tilde{p}_{i}^{\mu} \tilde{p}_{i}^{\nu}-n \cdot P^{\prime} \sum_{i} \frac{1}{\hat{p}_{i} \cdot n} \hat{p}_{i}^{\mu} \hat{p}_{i}^{\nu} .
$$

Substituting (2.4) and (2.5) into (1.7), we get,

$$
\begin{aligned}
B^{\mu \nu}= & -\frac{4 G^{2}}{R c^{7}}\left[\sum_{i=1}^{\tilde{n}} \sum_{\substack{j=1 \\
j \neq i}}^{\tilde{n}} \frac{\tilde{p}_{i} \cdot \tilde{p}_{j}}{\left\{\left(\tilde{p}_{i} \cdot \tilde{p}_{j}\right)^{2}-\tilde{p}_{i}^{2} \tilde{p}_{j}^{2}\right\}^{3 / 2}}\left\{\frac{3}{2} \tilde{p}_{i}^{2} \tilde{p}_{j}^{2}-\left(\tilde{p}_{i} \cdot \tilde{p}_{j}\right)^{2}\right\} \frac{\tilde{p}_{i}^{\mu}}{n \cdot \tilde{p}_{i}}\left(n \cdot \tilde{p}_{j} \tilde{p}_{i}^{\nu}-n \cdot \tilde{p}_{i} \tilde{p}_{j}^{\nu}\right)\right. \\
& \left.-\left\{\sum_{j=1}^{\tilde{n}} \tilde{p}_{j} \cdot n \sum_{i=1}^{\tilde{n}} \frac{1}{\tilde{p}_{i} \cdot n} \tilde{p}_{i}^{\mu} \tilde{p}_{i}^{\nu}-\sum_{j=1}^{m} p_{j}^{\prime} \cdot n \sum_{i=1}^{m} \frac{1}{p_{i}^{\prime} \cdot n} p_{i}^{\prime \mu} p_{i}^{\prime \nu}\right\}+\tilde{P}^{\mu} \tilde{P}^{\nu}-P^{\prime \mu} P^{\prime \nu}\right] .
\end{aligned}
$$

This reproduces (1.10). 


\section{Analysis of the subsubleading term}

We shall now rewrite the expression (1.8) for $F^{\mu \nu}$ by dividing the sum over final state momenta into the contribution from massless and massive particle momenta, denoted by $\hat{p}_{i}$ and $\tilde{p}_{i}$ respectively. $F_{(n)}^{\mu \nu}$ will denote contribution from terms where in each term, we have $n$ factors of $\hat{p}_{i}$. Therefore we have:

$$
\begin{aligned}
& F_{(0)}^{\mu \nu}=\frac{2 G^{3}}{R c^{11}}\left[4 \sum_{j=1}^{\tilde{n}} \tilde{p}_{j} \cdot n \sum_{\ell=1}^{\tilde{n}} \tilde{p}_{\ell} \cdot n \sum_{i=1}^{\tilde{n}} \frac{\tilde{p}_{i}^{\mu} \tilde{p}_{i}^{\nu}}{\tilde{p}_{i} \cdot n}-4 \sum_{j=1}^{m} p_{j}^{\prime} \cdot n \sum_{\ell=1}^{m} p_{\ell}^{\prime} \cdot n \sum_{i=1}^{m} \frac{p_{i}^{\prime \mu} p_{i}^{\prime \nu}}{p_{i}^{\prime} \cdot n}\right. \\
& +4 \sum_{\ell=1}^{\tilde{n}} \tilde{p}_{\ell} \cdot n \sum_{i=1}^{\tilde{n}} \sum_{\substack{j=1 \\
j \neq i}}^{\tilde{n}} \frac{1}{\tilde{p}_{i} \cdot n} \\
& \times \frac{\tilde{p}_{i} \cdot \tilde{p}_{j}}{\left\{\left(\tilde{p}_{i} \cdot \tilde{p}_{j}\right)^{2}-\tilde{p}_{i}^{2} \tilde{p}_{j}^{2}\right\}^{3 / 2}}\left\{2\left(\tilde{p}_{i} \cdot \tilde{p}_{j}\right)^{2}-3 \tilde{p}_{i}^{2} \tilde{p}_{j}^{2}\right\}\left\{n \cdot \tilde{p}_{j} \tilde{p}_{i}^{\mu} \tilde{p}_{i}^{\nu}-n \cdot \tilde{p}_{i} \tilde{p}_{i}^{\mu} \tilde{p}_{j}^{\nu}\right\} \\
& +2 \sum_{\ell=1}^{m} p_{\ell}^{\prime} \cdot n \sum_{i=1}^{m} \sum_{\substack{j=1 \\
j \neq i}}^{m} \frac{1}{p_{i}^{\prime} \cdot n} \\
& \times \frac{p_{i}^{\prime} \cdot p_{j}^{\prime}}{\left\{\left(p_{i}^{\prime} \cdot p_{j}^{\prime}\right)^{2}-p_{i}^{\prime 2} p_{j}^{\prime 2}\right\}^{3 / 2}}\left\{2\left(p_{i}^{\prime} \cdot p_{j}^{\prime}\right)^{2}-3 p_{i}^{\prime 2} p_{j}^{\prime 2}\right\}\left\{n \cdot p_{j}^{\prime} p_{i}^{\prime \mu} p_{i}^{\prime \nu}-n \cdot p_{i}^{\prime} p_{i}^{\prime \mu} p_{j}^{\prime \nu}\right\} \\
& +\sum_{i=1}^{\tilde{n}} \sum_{\substack{j=1 \\
j \neq i}}^{\tilde{n}} \sum_{\ell=1}^{\tilde{n}} \frac{1}{\tilde{p}_{i} \cdot n} \frac{\tilde{p}_{i} \cdot \tilde{p}_{j}}{\left\{\left(\tilde{p}_{i} \cdot \tilde{p}_{j}\right)^{2}-\tilde{p}_{i}^{2} \tilde{p}_{j}^{2}\right\}^{3 / 2}}\left\{2\left(\tilde{p}_{i} \cdot \tilde{p}_{j}\right)^{2}-3 \tilde{p}_{i}^{2} \tilde{p}_{j}^{2}\right\} \frac{\tilde{p}_{i} \cdot \tilde{p}_{\ell}}{\left\{\left(\tilde{p}_{i} \cdot \tilde{p}_{\ell}\right)^{2}-\tilde{p}_{i}^{2} \tilde{p}_{\ell}^{2}\right\}^{3 / 2}} \\
& \left.\times\left\{2\left(\tilde{p}_{i} \cdot \tilde{p}_{\ell}\right)^{2}-3 \tilde{p}_{i}^{2} \tilde{p}_{\ell}^{2}\right\}\left\{n . \tilde{p}_{j} \tilde{p}_{i}^{\mu}-n . \tilde{p}_{i} \tilde{p}_{j}^{\mu}\right\}\left\{n . \tilde{p}_{\ell} \tilde{p}_{i}^{\nu}-n . \tilde{p}_{i} \tilde{p}_{\ell}^{\nu}\right\}\right] .
\end{aligned}
$$

In writing down the expressions for $F_{(n)}^{\mu \nu}$ for $n \geq 1$, we shall try to simplify the sum over final state momenta using the relations:

$$
\sum_{j} \tilde{p}_{j}^{\mu}=\tilde{P}^{\mu}, \quad \sum_{j} \hat{p}_{j}^{\mu}=\left(P^{\prime}-\tilde{P}\right)^{\mu} .
$$

Another simplification follows from the observation that the expression $\left\{n \cdot p_{j} p_{i}^{\mu} p_{i}^{\nu}-\right.$ $\left.n . p_{i} p_{i}^{\mu} p_{j}^{\nu}\right\}$ vanishes for $j=i$. Therefore unless the factor multiplying it diverges for $j=i$, we can include in the sum over $j$ the term $j=i$ even if the original sum excludes this. This trick can often be used to make the sum over $i$ and $j$ into independent sums as long as either $i$ or $j$ represents a massless particle, since in this case we can first use (2.1) to replace the apparently divergent factor at $j=i$ by a finite term, and then include the contribution from the $j=i$ term in the sum. This gives:

$$
\begin{aligned}
F_{(1)}^{\mu \nu}= & \frac{2 G^{3}}{R c^{11}}\left[8 n \cdot\left(P^{\prime}-\tilde{P}\right) n \cdot \tilde{P} \sum_{i=1}^{\tilde{n}} \frac{\tilde{p}_{i}^{\mu} \tilde{p}_{i}^{\nu}}{\tilde{p}_{i} \cdot n}+4 n \cdot \tilde{P} n \cdot \tilde{P} \sum_{i} \frac{\hat{p}_{i}^{\mu} \hat{p}_{i}^{\nu}}{\hat{p}_{i} \cdot n}\right. \\
& +4 n \cdot\left(P^{\prime}-\tilde{P}\right) \sum_{i} \sum_{j \neq i} \frac{1}{\tilde{p}_{i} \cdot n} \\
& \times \frac{\tilde{p}_{i} \cdot \tilde{p}_{j}}{\left\{\left(\tilde{p}_{i} \cdot \tilde{p}_{j}\right)^{2}-\tilde{p}_{i}^{2} \tilde{p}_{j}^{2}\right\}^{3 / 2}}\left\{2\left(\tilde{p}_{i} \cdot \tilde{p}_{j}\right)^{2}-3 \tilde{p}_{i}^{2} \tilde{p}_{j}^{2}\right\}\left\{n \cdot \tilde{p}_{j} \tilde{p}_{i}^{\mu} \tilde{p}_{i}^{\nu}-n \cdot \tilde{p}_{i} \tilde{p}_{i}^{\mu} \tilde{p}_{j}^{\nu}\right\}
\end{aligned}
$$




$$
\begin{aligned}
& -8 n . \tilde{P} \sum_{i} \frac{1}{\tilde{p}_{i} \cdot n}\left\{n \cdot\left(P^{\prime}-\tilde{P}\right) \tilde{p}_{i}^{\mu} \tilde{p}_{i}^{\nu}-n \cdot \tilde{p}_{i} \tilde{p}_{i}^{(\mu}\left(P^{\prime}-\tilde{P}\right)^{\nu)}\right\} \\
& -8 n . \tilde{P} \sum_{i} \frac{1}{\hat{p}_{i} \cdot n}\left\{n \cdot \tilde{P} \hat{p}_{i}^{\mu} \hat{p}_{i}^{\nu}-n \cdot \hat{p}_{i} \hat{p}_{i}^{(\mu} \tilde{P}^{\nu)}\right\} \text { non } \\
& +4 \sum_{i} \frac{1}{\hat{p}_{i} \cdot n}\left\{n \cdot \tilde{P} \hat{p}_{i}^{\mu}-n \cdot \hat{p}_{i} \tilde{P}^{\mu}\right\}\left\{n . \tilde{P} \hat{p}_{i}^{\nu}-n \cdot \hat{p}_{i} \tilde{P}^{\nu}\right\} \\
& -4 \sum_{i} \sum_{j \neq i} \frac{1}{\tilde{p}_{i} \cdot n} \frac{\tilde{p}_{i} \cdot \tilde{p}_{j}}{\left\{\left(\tilde{p}_{i} \cdot \tilde{p}_{j}\right)^{2}-\tilde{p}_{i}^{2} \tilde{p}_{j}^{2}\right\}^{3 / 2}}\left\{2\left(\tilde{p}_{i} \cdot \tilde{p}_{j}\right)^{2}-3 \tilde{p}_{i}^{2} \tilde{p}_{j}^{2}\right\} \\
& \quad \times\left\{n \cdot \tilde{p}_{j} n \cdot\left(P^{\prime}-\tilde{P}\right) \tilde{p}_{i}^{\mu} \tilde{p}_{i}^{\nu}-n \cdot \tilde{p}_{i} n \cdot\left(P^{\prime}-\tilde{P}\right) \tilde{p}_{j}^{\mu} \tilde{p}_{i}^{\nu}-n \cdot \tilde{p}_{j} n \cdot \tilde{p}_{i} \tilde{p}_{i}^{(\mu}\left(P^{\prime}-\tilde{P}\right)^{\nu)}\right. \\
& \left.\left.\quad+\left(n \cdot \tilde{p}_{i}\right)^{2} \tilde{p}_{j}^{(\mu}\left(P^{\prime}-\tilde{P}\right)^{\nu)}\right\}\right] .
\end{aligned}
$$

This can be simplified to:

$$
\begin{aligned}
F_{(1)}^{\mu \nu}= & \frac{2 G^{3}}{R c^{11}}\left[4 n . \tilde{P}\left(P^{\prime}-\tilde{P}\right)^{\mu} \tilde{P}^{\nu}+4 n . \tilde{P}\left(P^{\prime}-\tilde{P}\right)^{\nu} \tilde{P}^{\mu}+4 n \cdot\left(P^{\prime}-\tilde{P}\right) \tilde{P}^{\mu} \tilde{P}^{\nu}\right. \\
- & -4 \sum_{i} \sum_{j \neq i} \frac{\tilde{p}_{i} \cdot \tilde{p}_{j}}{\left\{\left(\tilde{p}_{i} \cdot \tilde{p}_{j}\right)^{2}-\tilde{p}_{i}^{2} \tilde{p}_{j}^{2}\right\}^{3 / 2}}\left\{2\left(\tilde{p}_{i} \cdot \tilde{p}_{j}\right)^{2}-3 \tilde{p}_{i}^{2} \tilde{p}_{j}^{2}\right\} \\
& \left.\times\left\{-n \cdot \tilde{p}_{j} \tilde{p}_{i}^{(\mu}\left(P^{\prime}-\tilde{P}\right)^{\nu)}+n \cdot \tilde{p}_{i} \tilde{p}_{j}^{(\mu}\left(P^{\prime}-\tilde{P}\right)^{\nu)}\right\}\right]
\end{aligned}
$$

We now note that the last line is anti-symmetric under the exchange of $i$ and $j$ while the second line is symmetric under this exchange. Therefore this term vanishes after summing over $i$ and $j$, and we get:

$$
F_{(1)}^{\mu \nu}=\frac{2 G^{3}}{R c^{11}}\left[4 n . \tilde{P}\left(P^{\prime}-\tilde{P}\right)^{\mu} \tilde{P}^{\nu}+4 n . \tilde{P}\left(P^{\prime}-\tilde{P}\right)^{\nu} \tilde{P}^{\mu}+4 n .\left(P^{\prime}-\tilde{P}\right) \tilde{P}^{\mu} \tilde{P}^{\nu}\right] .
$$

We also have,

$$
\begin{aligned}
F_{(2)}^{\mu \nu}= & \frac{2 G^{3}}{R c^{11}}\left[4 n \cdot\left(P^{\prime}-\tilde{P}\right) n \cdot\left(P^{\prime}-\tilde{P}\right) \sum_{i=1}^{\tilde{n}} \frac{\tilde{p}_{i}^{\mu} \tilde{p}_{i}^{\nu}}{\tilde{p}_{i} \cdot n}+8 n \cdot\left(P^{\prime}-\tilde{P}\right) n \cdot \tilde{P} \sum_{i} \frac{\hat{p}_{i}^{\mu} \hat{p}_{i}^{\nu}}{\hat{p}_{i} \cdot n}\right. \\
& -8 n \cdot\left(P^{\prime}-\tilde{P}\right) \sum_{i} \frac{1}{\hat{p}_{i} \cdot n}\left\{n \cdot \tilde{P} \hat{p}_{i}^{\mu} \hat{p}_{i}^{\nu}-n \cdot \hat{p}_{i} \hat{p}_{i}^{\mu} \tilde{P}^{\nu}\right\} \\
& -8 n \cdot\left(P^{\prime}-\tilde{P}\right) \sum_{i} \frac{1}{\tilde{p}_{i} \cdot n}\left\{n \cdot\left(P^{\prime}-\tilde{P}\right) \tilde{p}_{i}^{\mu} \tilde{p}_{i}^{\nu}-n \cdot \tilde{p}_{i} \tilde{p}_{i}^{\mu}\left(P^{\prime}-\tilde{P}\right)^{\nu}\right\} \\
& -8 n \cdot \tilde{P} \sum_{i} \frac{1}{\hat{p}_{i} \cdot n}\left\{n \cdot\left(P^{\prime}-\tilde{P}\right) \hat{p}_{i}^{\mu} \hat{p}_{i}^{\nu}-n \cdot \hat{p}_{i} \hat{p}_{i}^{\mu}\left(P^{\prime}-\tilde{P}\right)^{\nu}\right\} \\
& +4 \sum_{i} \frac{1}{\tilde{p}_{i} \cdot n}\left\{n \cdot\left(P^{\prime}-\tilde{P}\right) n \cdot\left(P^{\prime}-\tilde{P}\right) \tilde{p}_{i}^{\mu} \tilde{p}_{i}^{\nu}-n \cdot \tilde{p}_{i} n \cdot\left(P^{\prime}-\tilde{P}\right)\left(P^{\prime}-\tilde{P}\right)^{\mu} \tilde{p}_{i}^{\nu}\right. \\
& \left.-n \cdot\left(P^{\prime}-\tilde{P}\right) n \cdot \tilde{p}_{i} \tilde{p}_{i}^{\mu}\left(P^{\prime}-\tilde{P}\right)^{\nu}+\left(n \cdot \tilde{p}_{i}\right)^{2}\left(P^{\prime}-\tilde{P}\right)^{\mu}\left(P^{\prime}-\tilde{P}\right)^{\nu}\right\} \\
& \left.+8 \sum_{i} \frac{1}{\hat{p}_{i} \cdot n}\left\{n \cdot\left(P^{\prime}-\tilde{P}\right) \hat{p}_{i}^{(\mu}-n \cdot \hat{p}_{i}\left(P^{\prime}-\tilde{P}\right)^{(\mu}\right\}\left\{n \cdot \tilde{P} \hat{p}_{i}^{\nu)}-n \cdot \hat{p}_{i} \tilde{P}^{\nu)}\right\}\right] .
\end{aligned}
$$


This can be simplified to:

$$
F_{(2)}^{\mu \nu}=\frac{2 G^{3}}{R c^{11}}\left[8 n \cdot\left(P^{\prime}-\tilde{P}\right)\left(P^{\prime}-\tilde{P}\right)^{(\mu} \tilde{P}^{\nu)}+4 n . \tilde{P}\left(P^{\prime}-\tilde{P}\right)^{\mu}\left(P^{\prime}-\tilde{P}\right)^{\nu}\right] .
$$

Finally we have,

$$
\begin{aligned}
F_{(3)}^{\mu \nu}= & \frac{2 G^{3}}{R c^{11}}\left[4 n \cdot\left(P^{\prime}-\tilde{P}\right) n \cdot\left(P^{\prime}-\tilde{P}\right) \sum_{i} \frac{\hat{p}_{i}^{\mu} \hat{p}_{i}^{\nu}}{\hat{p}_{i} \cdot n}\right. \\
& -8 n \cdot\left(P^{\prime}-\tilde{P}\right) \sum_{i} \frac{1}{\hat{p}_{i} \cdot n}\left\{n \cdot\left(P^{\prime}-\tilde{P}\right) \hat{p}_{i}^{\mu} \hat{p}_{i}^{\nu}-n \cdot \hat{p}_{i} \hat{p}_{i}^{\mu}\left(P^{\prime}-\tilde{P}\right)^{\nu}\right\} \\
& \left.+4 \sum_{i} \frac{1}{\hat{p}_{i} \cdot n}\left\{n \cdot\left(P^{\prime}-\tilde{P}\right) \hat{p}_{i}^{\mu}-n \cdot \hat{p}_{i}\left(P^{\prime}-\tilde{P}\right)^{\mu}\right\}\left\{n \cdot\left(P^{\prime}-\tilde{P}\right) \hat{p}_{i}^{\nu}-n \cdot \hat{p}_{i}\left(P^{\prime}-\tilde{P}\right)^{\nu}\right\}\right] .
\end{aligned}
$$

This can be simplified to:

$$
F_{(3)}^{\mu \nu}=\frac{2 G^{3}}{R c^{11}}\left[4 n \cdot\left(P^{\prime}-\tilde{P}\right)\left(P^{\prime}-\tilde{P}\right)^{\mu}\left(P^{\prime}-\tilde{P}\right)^{\nu}\right] .
$$

Using (3.6), (3.8) and (3.10), we get,

$$
F_{(1)}^{\mu \nu}+F_{(2)}^{\mu \nu}+F_{(3)}^{\mu \nu}=\frac{8 G^{3}}{R c^{11}}\left[n \cdot P^{\prime} P^{\prime \mu} P^{\prime \nu}-n \cdot \tilde{P} \tilde{P}^{\mu} \tilde{P}^{\nu}\right] .
$$

Adding this to (3.1) we get (1.12).

\section{Example involving scattering of massless particles}

In this section we shall compare our results to that of $[16,17]$ on the emission of soft gravitational radiation during the scattering of a pair of massless particles. For this comparison we shall set $c=1$ since the results of [17] were given in that convention. ${ }^{1}$ Let $\tilde{e}_{\mu \nu}(\omega, \vec{x})$ be the time Fourier transform of $e_{\mu \nu}(t, \vec{x})$ :

$$
\tilde{e}_{\mu \nu}(\omega, \vec{x})=\int d u e^{i \omega u} e_{\mu \nu}(t, \vec{x}), \quad u=t-t_{0} .
$$

Then $\tilde{e}_{\mu \nu}(\omega, \vec{x})$ has a small $\omega$ expansion of the form [3]:

$$
\tilde{e}_{\mu \nu}(\omega, \vec{x})=i A_{\mu \nu} \omega^{-1}-\left(B_{\mu \nu}-C_{\mu \nu}\right) \ln \omega+\frac{i}{2}\left(F_{\mu \nu}-G_{\mu \nu}\right) \omega(\ln \omega)^{2}+\cdots .,
$$

where $A_{\mu \nu}, B_{\mu \nu}, C_{\mu \nu}, F_{\mu \nu}$ and $G_{\mu \nu}$ are the same coefficients that appeared in the large $|u|$ expansion of $e_{\mu \nu}$. The $i \epsilon$ prescription inside $\ln \omega$ captures separate information on $B_{\mu \nu}$ and $C_{\mu \nu}$ and also on $F_{\mu \nu}$ and $G_{\mu \nu}$ [3], but at present we shall proceed ignoring the $i \epsilon$ prescription.

\footnotetext{
${ }^{1} \mathrm{~A}$ similar result was found in [18] where part of the contribution associated to the Coulomb phase of the soft radiation (e.g. the terms in the second line of (1.7)) was not included.
} 
When all the incoming and outgoing particles are massless, we get from (1.8), (1.9), (1.10) and (1.12),

$$
B^{\mu \nu}=-C^{\mu \nu}=-\frac{4 G^{2}}{R}\left[n \cdot P^{\prime} \sum_{i} \frac{p_{i}^{\prime \mu} p_{i}^{\prime \nu}}{n \cdot p_{i}^{\prime}}-P^{\prime \mu} P^{\prime \nu}\right],
$$

and

$$
F^{\mu \nu}=-G^{\mu \nu}=-\frac{16 G^{3}}{R} n \cdot P^{\prime}\left[n \cdot P^{\prime} \sum_{i} \frac{p_{i}^{\prime \mu} p_{i}^{\prime \nu}}{n \cdot p_{i}^{\prime}}-P^{\prime \mu} P^{\prime \nu}\right] .
$$

We also have, from (1.6),

$$
A^{\mu \nu}=\frac{2 G}{R}\left[-\sum_{i=1}^{n} p_{i}^{\mu} p_{i}^{\nu} \frac{1}{n \cdot p_{i}}+\sum_{i=1}^{m} p_{i}^{\prime \mu} p_{i}^{\prime \nu} \frac{1}{n \cdot p_{i}^{\prime}}\right] .
$$

We now apply these results to the specific case of scattering of two massless particles into two massless particles and soft gravitational radiation. Following [17], we label the momenta of the incoming and outgoing hard particles as:

$$
\begin{aligned}
& p_{1}^{\prime}=E(1,0,0,1), \quad p_{2}^{\prime}=E(1,0,0,-1), \\
& p_{1}=E\left(1, \sin \Theta_{s} \cos \phi, \sin \Theta_{s} \sin \phi, \cos \Theta_{s}\right), \\
& p_{2}=E\left(1,-\sin \Theta_{s} \cos \phi,-\sin \Theta_{s} \sin \phi,-\cos \Theta_{s}\right) .
\end{aligned}
$$

On the other hand, the direction of emission of the soft gravitational rediation, encoded in the four vector $n=(1, \hat{n})$, takes the form:

$$
n=(1, \sin \theta, 0, \cos \theta) .
$$

Our choice of frame is rotated by an angle $\phi$ about the $z$-axis relative to the frame used in [17], so that the direction of propagation of the soft gravitational wave, and not the momenta of the outgoing hard particles, lies in the $x$ - $z$ plane. We now define

$$
\hat{e}^{+}=(0, \cos \theta, i,-\sin \theta), \quad \hat{e}^{-}=(0, \cos \theta,-i,-\sin \theta),
$$

so that,

$$
\epsilon_{\mu \nu}^{+}=\frac{1}{2} \hat{e}_{\mu}^{+} \hat{e}_{\nu}^{+}, \quad \epsilon_{\mu \nu}^{-}=\frac{1}{2} \hat{e}_{\mu}^{-} \hat{e}_{\nu}^{-},
$$

denote left and right circular polarizations of soft gravitational waves traveling along $n$.

Using (4.2) we now get,

$$
\epsilon_{\mu \nu}^{ \pm} \tilde{e}^{\mu \nu}(\omega, \vec{x})=\epsilon_{\mu \nu}^{ \pm}\left[i A^{\mu \nu} \omega^{-1}-\left(B^{\mu \nu}-C^{\mu \nu}\right) \ln \omega+\frac{i}{2}\left(F^{\mu \nu}-G^{\mu \nu}\right) \omega(\ln \omega)^{2}+\cdots\right] .
$$

Since [17] gives the result for small $\theta$ and $\Theta_{s}$, we shall also make this approximation. However, in section 5 we have given the results for finite $\theta$ and $\Theta_{s}$. Now, for small $\theta, \Theta_{s}$,

$$
\begin{aligned}
& \hat{e}^{ \pm} \cdot p_{1}^{\prime}=-E \sin \theta \simeq-E \theta, \\
& \hat{e}^{ \pm} \cdot p_{2}^{\prime}=E \sin \theta \simeq E \theta,
\end{aligned}
$$




$$
\begin{aligned}
\hat{e}^{ \pm} \cdot p_{1} & =E\left(\sin \Theta_{s} \cos \theta \cos \phi-\cos \Theta_{s} \sin \theta \pm i \sin \Theta_{s} \sin \phi\right) \\
& \simeq E\left(\Theta_{s} \cos \phi-\theta \pm i \Theta_{s} \sin \phi\right), \\
\hat{e}^{ \pm} \cdot p_{2} & =-E\left(\sin \Theta_{s} \cos \theta \cos \phi-\cos \Theta_{s} \sin \theta \pm i \sin \Theta_{s} \sin \phi\right) \\
& \simeq-E\left(\Theta_{s} \cos \phi-\theta \pm i \Theta_{s} \sin \phi\right), \\
n \cdot p_{1}^{\prime} & =-E(1-\cos \theta) \simeq-E \theta^{2} / 2, \\
n \cdot p_{2}^{\prime} & =-E(1+\cos \theta) \simeq-2 E \\
n \cdot p_{1} & =-E\left(1-\sin \Theta_{s} \sin \theta \cos \phi-\cos \Theta_{s} \cos \theta\right) \simeq-E\left(\Theta_{s}^{2}+\theta^{2}-2 \Theta_{s} \theta \cos \phi\right) / 2 \\
n \cdot p_{2} & =-E\left(1+\sin \Theta_{s} \sin \theta \cos \phi+\cos \Theta_{s} \cos \theta\right) \simeq-2 E .
\end{aligned}
$$

It follows from these equations that $\epsilon_{\mu \nu}^{ \pm} p_{i}^{\mu} p_{i}^{\nu}$ and $\epsilon_{\mu \nu}^{ \pm} p_{i}^{\prime \mu} p_{i}^{\prime \nu}$ are quadratic in the small parameters $\Theta_{s}$ and $\theta$. Therefore only terms with $n . p_{1}$ or $n . p_{1}^{\prime}$ in the denominator will survive in this limit. This gives:

$$
\begin{aligned}
\epsilon_{\mu \nu}^{ \pm} A^{\mu \nu} & =\frac{G}{R}\left[-\frac{\hat{e}^{ \pm} \cdot p_{1} \hat{e}^{ \pm} \cdot p_{1}}{n \cdot p_{1}}+\frac{\hat{e}^{ \pm} \cdot p_{1}^{\prime} \hat{e}^{ \pm} \cdot p_{1}^{\prime}}{n \cdot p_{1}^{\prime}}\right] \\
& =\frac{2 G E}{R}\left[\frac{\left(\Theta_{s} \cos \phi-\theta \pm i \Theta_{s} \sin \phi\right)^{2}}{\left(\Theta_{s}^{2}+\theta^{2}-2 \Theta_{s} \theta \cos \phi\right)}-1\right], \\
\epsilon_{\mu \nu}^{ \pm}\left(B^{\mu \nu}-C^{\mu \nu}\right) & =-\frac{4 G^{2}}{R} n \cdot P^{\prime} \frac{\hat{e}^{ \pm} \cdot p_{1}^{\prime} \hat{e}^{ \pm} \cdot p_{1}^{\prime}}{n \cdot p_{1}^{\prime}}=-\frac{16 G^{2} E^{2}}{R},
\end{aligned}
$$

and

$$
\epsilon_{\mu \nu}^{ \pm}\left(F^{\mu \nu}-G^{\mu \nu}\right)=-\frac{16 G^{3}}{R}\left(n \cdot P^{\prime}\right)^{2} \frac{\hat{e}^{ \pm} \cdot p_{1}^{\prime} \hat{e}^{ \pm} \cdot p_{1}^{\prime}}{n \cdot p_{1}^{\prime}}=\frac{128 G^{3} E^{3}}{R} .
$$

Following [17], we introduce the variable $\psi$ via:

$$
\sin \psi=\frac{\Theta_{s} \sin \phi}{\left(\Theta_{s}^{2}+\theta^{2}-2 \Theta_{s} \theta \cos \phi\right)^{1 / 2}}, \quad \cos \psi=\frac{\Theta_{s} \cos \phi-\theta}{\left(\Theta_{s}^{2}+\theta^{2}-2 \Theta_{s} \theta \cos \phi\right)^{1 / 2}},
$$

so that (4.12) may be expressed as:

$$
\epsilon_{\mu \nu}^{ \pm} A^{\mu \nu}=\frac{2 G E}{R}\left[e^{ \pm 2 i \psi}-1\right] .
$$

Substituting (4.13), (4.14) and (4.16) into (4.10), we get,

$$
\epsilon_{\mu \nu}^{ \pm} \tilde{e}^{\mu \nu}=i \frac{2 G E}{R} \omega^{-1}\left[e^{ \pm 2 i \psi}-1-i 8 G E \omega \ln \omega+32 G^{2} E^{2} \omega^{2}(\ln \omega)^{2}\right] .
$$

Up to an overall normalization this agrees with the small $\omega$ expansion of eq. (6.20) of [17] after identifying the variable $R$ of [17], describing the Schwarzschild radius of the system, with $4 G E$. We shall now verify that the overall normalization also agrees.

To check the overall normalization, we compute the energy flux associated with (4.17) at the leading order in $\omega$. This can be done using the formula for the angular distribution of the energy flux with a given polarization. In the $8 \pi G=1$ unit the flux is given by (see e.g. [19]):

$$
\frac{d E_{ \pm}}{d \omega d \Omega}=\frac{\omega^{2}}{\pi} R^{2}\left|\epsilon_{\mu \nu}^{ \pm} \tilde{e}^{\mu \nu}\right|^{2}=\frac{E^{2}}{16 \pi^{3}}\left|e^{ \pm 2 i \psi}-1\right|^{2} .
$$


On the other hand, the same flux computed in [17] at the leading order in $\omega$ is given by (see eq. (6.13)):

$$
\frac{4 G E^{2}}{8 \pi^{2}}\left|e^{ \pm 2 i \psi}-1\right|^{2}=\frac{E^{2}}{16 \pi^{3}}\left|e^{ \pm 2 i \psi}-1\right|^{2} .
$$

Comparing (4.18) and (4.19) we see that the overall normalizations also match.

Even though we have derived the various formulæ in the limit of small $\Theta_{s}$ and $\theta$ for comparison with the results of [17], it follows from our general result that even for general values of $\Theta_{s}$ and $\theta$, our expressions for $B_{\mu \nu}=-C_{\mu \nu}$ and $F_{\mu \nu}=-G_{\mu \nu}$ remain the same as those given in (4.13) and (4.14). What is perhaps more striking is that even if the incoming states have a small enough impact parameter so that they form a black hole, possibly accompanied by hard radiation, the expressions for $B_{\mu \nu}$ and $F_{\mu \nu}$ do not change. This follows from the discussion in the last paragraph of section 1 since we have only one massive object in the final state.

Before concluding this section, we would like to discuss another aspect of the results given in [17]. [17] used the wave-form (4.17) to compute the total flux of soft radiation to subsubleading order. However on dimensional grounds, the $\ln \omega$ in (4.17) should be regarded as $\ln (\omega a)$ where $a$ is some scale of the order of the size of the scattering region, giving non universal contribution of order $\omega \ln a$. Therefore there could be terms of order $\omega$ inside the square bracket that have not been computed. This would give an additional contribution at the subleading order, spoiling the subsubleading results of [17]. It is easy to see that an imaginary term of order $\omega$ inside the square bracket in (4.17) will not contribute to the energy flux at the subleading order after summing over polarizations of the gravitational radiation. On the other hand a real term proportional to $\omega$ will violate the reality condition $\tilde{e}_{\mu \nu}(\omega)^{*}=\tilde{e}_{\mu \nu}(-\omega)$ that is required for the reality of the gravitational field. However there could be a contribution proportional to $\omega\{H(\omega)-H(-\omega)\}$ inside the square bracket, with $H$ denoting the Heaviside function, that satisfies the reality condition. If present, such a term would give subleading contribution to the energy flux, spoiling the result of [17].

We shall now show that such a term is absent, but for this we need to carefully keep track of the $i \epsilon$ prescription in the argument of the logarithms in (4.2). It follows from the analysis of [3], that at the subleading order, the time Fourier transform of the wave-form, including the $i \epsilon$ prescription, is given by:

$$
\left.-\frac{1}{2}\left\{B_{\mu \nu} \ln (\omega+i \epsilon)-C_{\mu \nu} \ln (\omega-i \epsilon)+B_{\mu \nu} \ln (-\omega-i \epsilon)-C_{\mu \nu} \ln (-\omega+i \epsilon)\right)\right\} .
$$

Using (4.3) this can be written as:

$$
\frac{1}{2} C_{\mu \nu}\{\ln (\omega+i \epsilon)+\ln (\omega-i \epsilon)+\ln (-\omega-i \epsilon)+\ln (-\omega+i \epsilon)\}=2 C_{\mu \nu} \ln |\omega| .
$$

Therefore at the subleading order we do not have terms proportional to $H(\omega)-H(-\omega)$. Note that this is a consequence of the relation $B_{\mu \nu}=-C_{\mu \nu}$ and seems to be present when all the incoming and outgoing particles are massless. From this it follows that (4.17) can be used to compute the total flux of energy carried by the gravitational radiation to order $\omega^{2}(\ln \omega)^{2}$, reproducing the result of [17]. 


\section{$5 \quad$ Energy flux from massless particle scattering}

In section 4 we compared our results for radiation during scattering of massless particles at small angle with those of [17]. In this section we shall compute the energy flux of low frequency gravitational radiation produced during such a scattering without making the small angle approximation.

Using (4.3)-(4.5) and (4.11) without making the small $\theta, \Theta_{s}$ approximation, we get

$$
\begin{aligned}
\epsilon_{\mu \nu}^{ \pm} A^{\mu \nu} & =\frac{2 G E}{R}\left[e^{ \pm 2 i \psi}-1\right], \\
\sin \psi & =\frac{\sin \Theta_{s} \sin \phi}{\left\{1-\left(\sin \Theta_{s} \sin \theta \cos \phi+\cos \Theta_{s} \cos \theta\right)^{2}\right\}^{1 / 2}}, \\
\cos \psi & =\frac{\sin \Theta_{s} \cos \theta \cos \phi-\cos \Theta_{s} \sin \theta}{\left\{1-\left(\sin \Theta_{s} \sin \theta \cos \phi+\cos \Theta_{s} \cos \theta\right)^{2}\right\}^{1 / 2}}, \\
\epsilon_{\mu \nu}^{ \pm}\left(B^{\mu \nu}-C^{\mu \nu}\right) & =-\frac{16 G^{2} E^{2}}{R}, \quad \epsilon_{\mu \nu}^{ \pm}\left(F^{\mu \nu}-G^{\mu \nu}\right)=\frac{128 G^{3} E^{3}}{R} .
\end{aligned}
$$

Using (4.2) and the first equality in (4.18), and summing over polarizations, we now get the total differential flux in the $8 \pi G=1$ unit:

$$
\begin{aligned}
\frac{d E}{d \omega d \Omega} & \equiv \sum_{ \pm} \frac{d E_{ \pm}}{d \omega d \Omega} \\
& =\sum_{ \pm} \frac{\omega^{2}}{\pi} R^{2}\left|\epsilon_{\mu \nu}^{ \pm}\left\{i A^{\mu \nu} \omega^{-1}-\ln \omega\left(B^{\mu \nu}-C^{\mu \nu}\right)+\frac{i}{2} \omega(\ln \omega)^{2}\left(F^{\mu \nu}-G^{\mu \nu}\right)\right\}\right|^{2}
\end{aligned}
$$

Using (5.1), (5.1), this can be written as

$$
P(\theta, \phi)+Q(\theta, \phi) \omega^{2}(\ln \omega)^{2}+\mathcal{O}\left(\omega^{2} \ln \omega\right),
$$

where

$$
P(\theta, \phi)=\frac{E^{2}}{2 \pi^{3}} \sin ^{2} \psi, \quad Q(\theta, \phi)=\frac{E^{4}}{8 \pi^{5}}\left(1-2 \sin ^{2} \psi\right) .
$$

Therefore for computing the total flux, we need to evaluate the integral:

$$
\begin{aligned}
I & \equiv \int_{0}^{\pi} \sin \theta d \theta \int_{0}^{2 \pi} d \phi \sin ^{2} \psi \\
& =\int_{0}^{\pi} \sin \theta d \theta \int_{0}^{2 \pi} d \phi \frac{\sin ^{2} \Theta_{s} \sin ^{2} \phi}{\left\{1-\left(\sin \Theta_{s} \sin \theta \cos \phi+\cos \Theta_{s} \cos \theta\right)^{2}\right\}} .
\end{aligned}
$$

Now, writing

$$
\begin{aligned}
\frac{\sin ^{2} \Theta_{s} \sin ^{2} \phi}{\left\{1-\left(\sin \Theta_{s} \sin \theta \cos \phi+\cos \Theta_{s} \cos \theta\right)^{2}\right\}}= & \frac{1}{2} \frac{\sin ^{2} \Theta_{s} \sin ^{2} \phi}{\left\{1-\left(\sin \Theta_{s} \sin \theta \cos \phi+\cos \Theta_{s} \cos \theta\right)\right\}} \\
& +(\theta \rightarrow \pi-\theta, \phi \rightarrow \phi+\pi),
\end{aligned}
$$

and noting that both terms produce the same integral, we can express $I$ as:

$$
I=\int_{0}^{\pi} \sin \theta d \theta \int_{0}^{2 \pi} d \phi \frac{\sin ^{2} \Theta_{s} \sin ^{2} \phi}{\left\{1-\left(\sin \Theta_{s} \sin \theta \cos \phi+\cos \Theta_{s} \cos \theta\right)\right\}} .
$$


We can carry out the $\phi$ integral by defining $z=e^{i \phi}$, replacing $\sin \phi$ by $\left(z-z^{-1}\right) /(2 i) \cos \phi$ by $\left(z+z^{-1}\right) / 2$, and regarding (5.7) as a contour integral over $z$ along the unit circle. The resulting integrand has double pole at $z=0$ and single poles at $z=z_{ \pm}$where

$$
z_{ \pm}=\frac{\left(1 \pm \cos \theta_{s}\right)(1 \mp \cos \theta)}{\sin \theta \sin \theta_{s}}
$$

It is easy to see that for $\theta>\Theta_{s}, z_{+}>1, z_{-}<1$ and for $\theta<\Theta_{s}, z_{+}<1, z_{-}>1$. We can now perform the contour integral by picking up the residues at the poles inside the unit circle. The result is:

$$
I=2 \pi \int_{0}^{\pi} \sin \theta d \theta\left[H\left(\Theta_{s}-\theta\right) \frac{1+\cos \Theta_{s}}{1+\cos \theta}+H\left(\theta-\Theta_{s}\right) \frac{1-\cos \Theta_{s}}{1-\cos \theta}\right],
$$

where $H$ is the step function. This arises due to the fact that as $\theta$ varies from being below $\Theta_{s}$ to above $\Theta_{s}$, the poles of the integrand move across the integration contour. After carrying out the $\theta$ integration we get:

$$
I=2 \pi\left[2 \ln 2-\left(1+\cos \Theta_{s}\right) \ln \left(1+\cos \Theta_{s}\right)-\left(1-\cos \Theta_{s}\right) \ln \left(1-\cos \Theta_{s}\right)\right] .
$$

We can now use (5.4) to calculate the energy flux integrated over all angles, up to order $\omega^{2}(\ln \omega)^{2}$ :

$$
\begin{aligned}
\int \frac{d E}{d \omega d \Omega} d \Omega= & \int_{0}^{\pi} \sin \theta d \theta \int_{0}^{2 \pi} d \phi\left[P(\theta, \phi)+Q(\theta, \phi) \omega^{2}(\ln \omega)^{2}\right] \\
= & \frac{E^{2}}{\pi^{2}}\left[2 \ln 2-\left(1+\cos \Theta_{s}\right) \ln \left(1+\cos \Theta_{s}\right)-\left(1-\cos \Theta_{s}\right) \ln \left(1-\cos \Theta_{s}\right)\right] \\
& +\frac{E^{4}}{2 \pi^{4}} \omega^{2}(\ln \omega)^{2}[1-2 \ln 2 \\
& \left.+\left(1+\cos \Theta_{s}\right) \ln \left(1+\cos \Theta_{s}\right)+\left(1-\cos \Theta_{s}\right) \ln \left(1-\cos \Theta_{s}\right)\right]
\end{aligned}
$$

The small $\Theta_{s}$ expansion of this function takes the form:

$$
\begin{aligned}
\int \frac{d E}{d \omega d \Omega} d \Omega= & \frac{E^{2}}{\pi^{2}} \frac{\Theta_{s}^{2}}{2}\left\{1+2 \ln 2+\ln \Theta_{s}^{-2}+\mathcal{O}\left(\Theta_{s}^{3}\right)\right\} \\
& +\frac{E^{4}}{2 \pi^{4}} \omega^{2}(\ln \omega)^{2}\left[1-\frac{\Theta_{s}^{2}}{2}\left\{1+2 \ln 2+\ln \Theta_{s}^{-2}\right\}+\mathcal{O}\left(\Theta_{s}^{3}\right)\right]+\mathcal{O}\left(\omega^{2} \ln \omega\right) .
\end{aligned}
$$

Even though we have evaluated (5.11) for general $\Theta_{s}$, during this derivation we have ignored the possible modification of $A_{\mu \nu}$ due to radiation emitted during the scattering. Now for scattering at large impact parameter $b$, we have $\Theta_{s} \sim E / b$ [20]. If we assume that the spectrum of gravitational radiation falls off rapidly for $\omega>b^{-1} \sim \Theta_{s} / E$, then integrating (5.12) in the range $0 \leq \omega \leq \Theta_{s} / E$ we see that the total radiated energy during the scattering is of order $E \Theta_{s}^{3}$ times possible factors of $\ln \Theta_{s}^{-1}$. Since the correction to $\epsilon_{\mu \nu}^{ \pm} A^{\mu \nu}$ from a final state particle is proportional to the energy carried by the particle, we see that $\epsilon_{\mu \nu}^{ \pm} A^{\mu \nu}$ can receive correction of order $E \Theta_{s}^{3}$, and this in turn can affect the coefficients appearing in (5.11) by terms of order $\Theta_{s}^{3}$. However expansion up to order $\Theta_{s}^{2}$, given in (5.12) can be trusted. 
It was shown in [16] however that in the near forward direction, the actual cut-off on $\omega$ extends beyond $1 / b$ and as a result the net energy of emitted radiation is of order $\Theta_{s}^{2}$ with possible logarithmic corrections. Therefore one might worry that this will give corrections to $\epsilon_{\mu \nu}^{ \pm} A^{\mu \nu}$ of order $\Theta_{s}^{2}$ and affect the order $\Theta_{s}^{2}$ coefficient of the $\omega^{2}(\ln \omega)^{2}$ term. However one can see as follows that this is not the case. If the energy is emitted strictly in the forward direction, then it effectively amounts to a redistribution of the energy among the various final state particles in the forward direction and does not affect $A_{\mu \nu}$. For example a final state particle of momentum $\lambda p$ and another particle of momentum $(1-\lambda) p$ give the same contribution to $A_{\mu \nu}$ as a single particle of momentum $p$. Therefore the relevant correction to $A_{\mu \nu}$ can be estimated from the difference between the emission in the forward direction and in a direction making an angle of order $\delta \Theta_{s} \sim \Theta_{s}$ with the forward direction. We see from (5.1) that when we change $\Theta_{s}$ by $\delta \Theta_{s}$, the change in $\epsilon_{\mu \nu}^{ \pm} A^{\mu \nu}$ is of order $E \delta \Theta_{s}$ even when $\delta \Theta_{s} \sim \Theta_{s}$ but $\theta$ is finite, but is of order $E$ when $\theta$ and $\delta \Theta_{s}$ are both of order $\Theta_{s}$. Using $E \sim \Theta_{s}^{2}$ and $\delta \Theta_{s} \sim \Theta_{s}$, one finds that for finite $\theta$, the correction to $\epsilon_{\mu \nu}^{ \pm} A^{\mu \nu}$ is still of order $\Theta_{s}^{3}$ (possibly multiplied by powers of $\ln \Theta_{s}^{-1}$ ) while for $\theta \sim \Theta_{s}$ the correction to $\epsilon_{\mu \nu}^{ \pm} A^{\mu \nu}$ is of order $\Theta_{s}^{2}$. Taking into account the extra factor of $\Theta_{s}^{2}$ that we get when $\theta$ integration is restricted to be of order $\Theta_{s}$, we see that the correction to (5.11) due to the modification of $A_{\mu \nu}$ by the final state radiation is of order $\Theta_{s}^{3}$ times possible logarithmic corrections.

The positivity of the coefficient of the $\omega^{2}(\ln \omega)^{2}$ term for small $\Theta_{s}$ shows that the flux has a local minimum at $\omega=0$ and therefore has a maximum elsewhere, presumably around $\omega \sim b^{-1}$. This confirms the prediction of [17]. The actual coefficient of this term differs from that of [17] by a factor of 2 in the small $\Theta_{s}$ limit. On the other hand the coefficient of the $E^{2} \Theta_{s}^{2} /\left(2 \pi^{2}\right)$ term differs from that of [17] by the additive constant $2 \ln 2$. Both of these can be attributed to the fact that [17] computed the differential flux in the small $\theta$ approximation, whereas (5.12) receives contribution also from the finite $\theta$ region.

\section{Discussion}

In this paper we have observed that the late time radiation during a scattering process is independent of the final state massless particle momenta. A similar phenomenon has now also been observed for the spin dependent part of the final state radiation [21]. These results are based on the analysis of the general formula for the radiation tail, but we do not know of any deeper explanation. The usual explanation of soft theorems based on the analysis of asymptotic symmetries does not work for logarithmic corrections. Some attempts to modify the asymptotic symmetry analysis to include the logarithmic corrections has been made in [22-25] for electrodynamics. Possible generalization of these results to gravity could give some insight into the phenomena described here.

We also observed that for a binary black-hole merger process the gravitational tail memories at order $u^{-1}, u^{-2} \ln u$ vanish when we treat the inspiral binary as a singe initial bound state object which decays to one massive black-hole and lots of massless radiation. A similar vanishing phenomena for spin dependent gravitational tail memory at order $u^{-2}$ has also been observed in [21]. Vanishing of the late time gravitational waveform at future null infinity is consistent with the prediction from Price's law for nearly spherical gravitational 
collapse $[26-28] .{ }^{2}$ Late time behaviour of scalar perturbation near future time-like and null hyper-surfaces has been widely studied in a spherically symmetric background to determine Price's law for scalar fluctuation [26, 29-35]. According to our current understanding a nonlinear version of Price's law for gravitational fluctuation has not been studied rigorously so far. In that case our observation on the vanishing of gravitational tail memories at the orders described above can be thought of as new predictions for a non-linear version of Price's law for gravitational fluctuation.

We have restricted our analysis to four dimensions, but one could ask if similar results may hold in higher dimensions. It is known that due to phase space suppression in the Fourier integral, the leading soft term in the amplitude proportional to $1 / \omega$ does not produce a late time memory effect in the final state radiation. Furthermore, the infrared effects are suppressed in higher dimensions and do not generate logarithmic corrections at low orders. However it is possible that they would generate $\omega^{n} \ln \omega$ type of terms in the Fourier transform of the final state radiation with high power of $n$. As these are nonanalytic despite having a finite $\omega \rightarrow 0$ limit, these could give rise to late time tail in the retarded time with high power $1 / u$. It will be interesting to explore whether the coefficient of these tail terms enjoy the same cancelation properties as described in this paper.

\section{Acknowledgments}

We wish to thank Dimitri Colferai and Gabriele Veneziano for useful discussions, comments on an earlier version of the draft and encouraging us to do the detailed analysis described in section 5. We thank the anonymous referee for suggesting improvements on the paper. B.S. is supported by the Simons Foundation grant 488649 (Simons Collaboration on the Nonperturbative Bootstrap) and by the Swiss National Science Foundation through the National Centre of Competence in Research SwissMAP. The work of A.S. was supported by the Infosys chair professorship and the J. C. Bose fellowship of the Department of Science and Technology, India. We want to thank the organizers and participants of the workshop "Gravitational scattering, inspiral, and radiation" at the Galileo Galilei Institute for Theoretical Physics in Florence, for hospitality and fruitful exchanges.

\section{A Effect of electromagnetic interaction}

If the incoming and the outgoing particles carry electric charge then the coefficients $B_{\mu \nu}$, $C_{\mu \nu}, F_{\mu \nu}$ and $G_{\mu \nu}$ receive additional corrections [2-4]. In this appendix we shall show that even in the presence of these corrections, $B_{\mu \nu}$ and $F_{\mu \nu}$ continue to be independent of the momenta (and charges) carried by individual massless particles in the final state.

The extra contribution to $B_{\mu \nu}$ due to electromagnetic interaction is given by [3]:

$$
\Delta B^{\mu \nu}=-\frac{2 G}{R c^{5}}\left[\sum_{\substack { i=1 \\
\begin{subarray}{c}{n \\
j \neq i{ i = 1 \\
\begin{subarray} { c } { n \\
j \neq i } }\end{subarray}}^{n} \frac{1}{\left\{\left(p_{i} \cdot p_{j}\right)^{2}-p_{i}^{2} p_{j}^{2}\right\}^{3 / 2}} \frac{p_{i}^{\mu}}{n \cdot p_{i}}\left(n \cdot p_{j} p_{i}^{\nu}-n \cdot p_{i} p_{j}^{\nu}\right) \times \frac{1}{4 \pi} q_{i} q_{j} p_{i}^{2} p_{j}^{2}\right],
$$

\footnotetext{
${ }^{2}$ We wish to thank Luc Blanchet and Thibault Damour for pointing out possible connection between our results and Price's law.
} 
where $q_{i}$ is the charge carrried by the $i$-th final state particles, in units where the electrostatic force between a pair of charges separated by distance $r$ is given by $q_{i} q_{j} /\left(4 \pi r^{2}\right)$. (A.1) clearly vanishes if either $i$ or $j$ represents a massless particle.

The correction to $F^{\mu \nu}$ is given by [4]:

$$
\begin{aligned}
& \Delta F^{\mu \nu}=\frac{2 G}{R c^{9}}\left[-4 G \sum_{\ell=1}^{n} p_{\ell} \cdot n \sum_{i=1}^{n} \sum_{\substack{j=1 \\
j \neq i}}^{n} \frac{1}{p_{i} \cdot n}\right. \\
& \times \frac{1}{\left\{\left(p_{i} \cdot p_{j}\right)^{2}-p_{i}^{2} p_{j}^{2}\right\}^{3 / 2}}\left\{n \cdot p_{j} p_{i}^{\mu} p_{i}^{\nu}-n \cdot p_{i} p_{i}^{\mu} p_{j}^{\nu}\right\} \times \frac{q_{i} q_{j}}{4 \pi} p_{i}^{2} p_{j}^{2} \\
& -2 G \sum_{\ell=1}^{m} p_{\ell}^{\prime} \cdot n \sum_{i=1}^{m} \sum_{\substack{j=1 \\
j \neq i}}^{m} \frac{1}{p_{i}^{\prime} \cdot n} \\
& \times \frac{1}{\left\{\left(p_{i}^{\prime} \cdot p_{j}^{\prime}\right)^{2}-p_{i}^{\prime 2} p_{j}^{\prime 2}\right\}^{3 / 2}}\left\{n \cdot p_{j}^{\prime} p_{i}^{\prime \mu} p_{i}^{\prime \nu}-n \cdot p_{i}^{\prime} p_{i}^{\prime \mu} p_{j}^{\prime \nu}\right\} \times \frac{q_{i}^{\prime} q_{j}^{\prime}}{4 \pi} p_{i}^{\prime 2} p_{j}^{\prime 2} \\
& -2 G \sum_{i=1}^{n} \sum_{\substack{j=1 \\
j \neq i}}^{n} \sum_{\ell=1}^{n} \frac{1}{p_{i} . n} \frac{p_{i} \cdot p_{j}}{\left\{\left(p_{i} \cdot p_{j}\right)^{2}-p_{i}^{2} p_{j}^{2}\right\}^{3 / 2}}\left\{2\left(p_{i} \cdot p_{j}\right)^{2}-3 p_{i}^{2} p_{j}^{2}\right\} \\
& \times \frac{1}{\left\{\left(p_{i} \cdot p_{\ell}\right)^{2}-p_{i}^{2} p_{\ell}^{2}\right\}^{3 / 2}} \times \frac{q_{i} q_{\ell}}{4 \pi} p_{i}^{2} p_{\ell}^{2}\left\{n \cdot p_{j} p_{i}^{\mu}-n \cdot p_{i} p_{j}^{\mu}\right\}\left\{n \cdot p_{\ell} p_{i}^{\nu}-n \cdot p_{i} p_{\ell}^{\nu}\right\} \\
& +c^{2} \sum_{i=1}^{n} \sum_{\substack{j=1 \\
j \neq i}}^{n} \sum_{\substack{\ell=1 \\
\ell \neq i}}^{n} \frac{1}{p_{i} \cdot n} \frac{1}{\left\{\left(p_{i} \cdot p_{j}\right)^{2}-p_{i}^{2} p_{j}^{2}\right\}^{3 / 2}} \frac{1}{\left\{\left(p_{i} \cdot p_{\ell}\right)^{2}-p_{i}^{2} p_{\ell}^{2}\right\}^{3 / 2}} \\
& \left.\times \frac{q_{i} q_{j}}{4 \pi} p_{i}^{2} p_{j}^{2} \times \frac{q_{i} q_{\ell}}{4 \pi} p_{i}^{2} p_{\ell}^{2}\left\{n \cdot p_{j} p_{i}^{\mu}-n \cdot p_{i} p_{j}^{\mu}\right\}\left\{n \cdot p_{\ell} p_{i}^{\nu}-n \cdot p_{i} p_{\ell}^{\nu}\right\}\right] .
\end{aligned}
$$

It is understood that the expression needs to be symmetrized under the exchange of $\mu$ and $\nu$. As before we divide the final state particles into massive particles carrying momenta $\tilde{p}_{i}$ and charges $\tilde{q}_{i}$ and massless particles with momenta $\hat{p}_{i}$ and charges $\hat{q}_{i}$. Examining this expression we see that the only contributions from massless final state particles can come when $p_{\ell}$ represents a massless particle momentum in the first term inside the square bracket and when $p_{j}$ represents a massless particle momentum in the third term inside the square bracket. The first contribution may be expressed as:

$$
-4 G \sum_{\ell} \hat{p}_{\ell} \cdot n \sum_{i} \sum_{\substack{j \\ j \neq i}} \frac{1}{\tilde{p}_{i} \cdot n} \frac{1}{\left\{\left(\tilde{p}_{i} \cdot \tilde{p}_{j}\right)^{2}-\tilde{p}_{i}^{2} \tilde{p}_{j}^{2}\right\}^{3 / 2}}\left\{n \cdot \tilde{p}_{j} \tilde{p}_{i}^{\mu} \tilde{p}_{i}^{\nu}-n . \tilde{p}_{i} \tilde{p}_{i}^{\mu} \tilde{p}_{j}^{\nu}\right\} \times \frac{\tilde{q}_{i} \tilde{q}_{j}}{4 \pi} \tilde{p}_{i}^{2} \tilde{p}_{j}^{2} .
$$

On the other hand when the sum over $j$ runs over massless particles in the third term within the square bracket, the contribution takes the form:

$$
\begin{aligned}
4 G \sum_{j} \sum_{i} & \sum_{\substack{\ell \\
\ell \neq i}} \frac{1}{\tilde{p}_{i} \cdot n} \frac{1}{\left\{\left(\tilde{p}_{i} \cdot \tilde{p}_{\ell}\right)^{2}-\tilde{p}_{i}^{2} \tilde{p}_{\ell}^{2}\right\}^{3 / 2}} \\
& \times \frac{\tilde{q}_{i} \tilde{q}_{\ell}}{4 \pi} \tilde{p}_{i}^{2} \tilde{p}_{\ell}^{2}\left\{n \cdot \hat{p}_{j} \tilde{p}_{i}^{\mu}-n \cdot \tilde{p}_{i} \hat{p}_{j}^{\mu}\right\}\left\{n \cdot \tilde{p}_{\ell} \tilde{p}_{i}^{\nu}-n \cdot \tilde{p}_{i} \tilde{p}_{\ell}^{\nu}\right\} .
\end{aligned}
$$


In the above expression, relabelling $\ell$ as $j$ and $j$ as $\ell$ and simplifying we get:

$$
\begin{aligned}
4 G \sum_{\ell} \sum_{i} \sum_{\substack{j \\
j \neq i}} \frac{1}{\tilde{p}_{i} \cdot n} \frac{1}{\left\{\left(\tilde{p}_{i} \cdot \tilde{p}_{j}\right)^{2}-\tilde{p}_{i}^{2} \tilde{p}_{j}^{2}\right\}^{3 / 2}} & \\
& \quad \times \frac{\tilde{q}_{i} \tilde{q}_{j}}{4 \pi} \tilde{p}_{i}^{2} \tilde{p}_{j}^{2}\left\{n \cdot \hat{p}_{\ell} \tilde{p}_{i}^{\mu}-n \cdot \tilde{p}_{i} \hat{p}_{\ell}^{\mu}\right\}\left\{n \cdot \tilde{p}_{j} \tilde{p}_{i}^{\nu}-n \cdot \tilde{p}_{i} \tilde{p}_{j}^{\nu}\right\} \\
= & 4 G \sum_{\ell} \hat{p}_{\ell} \cdot n \sum_{i} \sum_{\substack{j \\
j \neq i}} \frac{1}{\tilde{p}_{i} \cdot n} \frac{1}{\left\{\left(\tilde{p}_{i} \cdot \tilde{p}_{j}\right)^{2}-\tilde{p}_{i}^{2} \tilde{p}_{j}^{2}\right\}^{3 / 2}}\left\{n \cdot \tilde{p}_{j} \tilde{p}_{i}^{\mu} \tilde{p}_{i}^{\nu}-n \cdot \tilde{p}_{i} \tilde{p}_{i}^{\mu} \tilde{p}_{j}^{\nu}\right\} \times \frac{\tilde{q}_{i} \tilde{q}_{j}}{4 \pi} \tilde{p}_{i}^{2} \tilde{p}_{j}^{2} \\
& -4 G \sum_{\ell} \sum_{i} \sum_{\substack{j \\
j \neq i}} \frac{1}{\left\{\left(\tilde{p}_{i} \cdot \tilde{p}_{j}\right)^{2}-\tilde{p}_{i}^{2} \tilde{p}_{j}^{2}\right\}^{3 / 2}} \hat{p}_{\ell}^{\mu}\left\{n \cdot \tilde{p}_{j} \tilde{p}_{i}^{\nu}-n \cdot \tilde{p}_{i} \tilde{p}_{j}^{\nu}\right\} \times \frac{\tilde{q}_{i} \tilde{q}_{j}}{4 \pi} \tilde{p}_{i}^{2} \tilde{p}_{j}^{2} .
\end{aligned}
$$

We now see that the first term on the right hand side of (A.5) cancels (A.3) and the second term vanishes since the summand is anti-symmetric under the exchange of $i$ and $j$. Hence we do not get any contribution involving final state massless particles due to long-range electromagnetic interaction.

Open Access. This article is distributed under the terms of the Creative Commons Attribution License (CC-BY 4.0), which permits any use, distribution and reproduction in any medium, provided the original author(s) and source are credited.

\section{References}

[1] A. Laddha and A. Sen, Observational signature of the logarithmic terms in the soft graviton theorem, Phys. Rev. D 100 (2019) 024009 [arXiv: 1806.01872] [INSPIRE].

[2] B. Sahoo and A. Sen, Classical and quantum results on logarithmic terms in the soft theorem in four dimensions, JHEP 02 (2019) 086 [arXiv: 1808.03288] [INSPIRE].

[3] A.P. Saha, B. Sahoo and A. Sen, Proof of the classical soft graviton theorem in D =4, JHEP 06 (2020) 153 [arXiv: 1912.06413] [INSPIRE].

[4] B. Sahoo, Classical sub-subleading soft photon and soft graviton theorems in four spacetime dimensions, JHEP 12 (2020) 070 [arXiv:2008.04376] [INSPIRE].

[5] Y.B. Zel'dovich and A.G. Polnarev, Radiation of gravitational waves by a cluster of superdense stars, Sov. Astron. 18 (1974) 17 [INSPIRE].

[6] V.B. Braginsky and L.P. Grishchuk, Kinematic resonance and memory effect in free mass gravitational antennas, Sov. Phys. JETP 62 (1985) 427 [Zh. Eksp. Teor. Fiz. 89 (1985) 744] [INSPIRE].

[7] V.B. Braginsky and K.S. Thorne, Gravitational-wave bursts with memory and experimental prospects, Nature 327 (1987) 123.

[8] M. Ludvigsen, Geodesic deviation at null infinity and the physical effects of very long wave gravitational radiation, Gen. Rel. Grav. 21 (1989) 1205 [INSPIRE].

[9] D. Christodoulou, Nonlinear nature of gravitation and gravitational wave experiments, Phys. Rev. Lett. 67 (1991) 1486 [InSPIRE].

[10] K.S. Thorne, Gravitational-wave bursts with memory: the Christodoulou effect, Phys. Rev. D 45 (1992) 520 [INSPIRE]. 
[11] L. Blanchet and T. Damour, Hereditary effects in gravitational radiation, Phys. Rev. D 46 (1992) 4304 [INSPIRE].

[12] M. Favata, The gravitational-wave memory effect, Class. Quant. Grav. 27 (2010) 084036 [arXiv: 1003.3486] [INSPIRE].

[13] A. Tolish and R.M. Wald, Retarded fields of null particles and the memory effect, Phys. Rev. D 89 (2014) 064008 [arXiv:1401.5831] [INSPIRE].

[14] L. Bieri and D. Garfinkle, Perturbative and gauge invariant treatment of gravitational wave memory, Phys. Rev. D 89 (2014) 084039 [arXiv: 1312.6871] [InSPIRE].

[15] A. Strominger and A. Zhiboedov, Gravitational memory, BMS supertranslations and soft theorems, JHEP 01 (2016) 086 [arXiv:1411.5745] [INSPIRE].

[16] A. Gruzinov and G. Veneziano, Gravitational radiation from massless particle collisions, Class. Quant. Grav. 33 (2016) 125012 [arXiv:1409.4555] [INSPIRE].

[17] M. Ciafaloni, D. Colferai and G. Veneziano, Infrared features of gravitational scattering and radiation in the eikonal approach, Phys. Rev. D 99 (2019) 066008 [arXiv:1812.08137] [INSPIRE].

[18] A. Addazi, M. Bianchi and G. Veneziano, Soft gravitational radiation from ultra-relativistic collisions at sub- and sub-sub-leading order, JHEP 05 (2019) 050 [arXiv:1901.10986] [INSPIRE].

[19] A. Laddha and A. Sen, Gravity waves from soft theorem in general dimensions, JHEP 09 (2018) 105 [arXiv: 1801.07719] [INSPIRE].

[20] D. Amati, M. Ciafaloni and G. Veneziano, Superstring collisions at Planckian energies, Phys. Lett. B 197 (1987) 81 [INSPIRE].

[21] D. Ghosh and B. Sahoo, Spin dependent gravitational tail memory in $D=4$, arXiv:2106.10741 [INSPIRE].

[22] M. Campiglia and A. Laddha, Loop corrected soft photon theorem as a Ward identity, JHEP 10 (2019) 287 [arXiv: 1903.09133] [InSPIRE].

[23] S. Atul Bhatkar, Ward identity for loop level soft photon theorem for massless QED coupled to gravity, JHEP 10 (2020) 110 [arXiv:1912.10229] [INSPIRE].

[24] S. Atul Bhatkar, New asymptotic conservation laws forelectromagnetism, JHEP 02 (2021) 082 [arXiv : 2007.03627] [INSPIRE].

[25] S. Atul Bhatkar, Asymptotic conservation law with Feynman boundary condition, Phys. Rev. D 103 (2021) 125026 [arXiv:2101.09734] [InSPIRE].

[26] R.H. Price, Nonspherical perturbations of relativistic gravitational collapse. 1. Scalar and gravitational perturbations, Phys. Rev. D 5 (1972) 2419 [INSPIRE].

[27] R.H. Price, Nonspherical perturbations of relativistic gravitational collapse. Part II. Integer-spin, zero-rest-mass fields, Phys. Rev. D 5 (1972) 2439 [INSPIRE].

[28] S. Ma and L. Zhang, Price's law for spin fields on a Schwarzschild background, arXiv: 2104.13809 [INSPIRE].

[29] C. Gundlach, R.H. Price and J. Pullin, Late time behavior of stellar collapse and explosions: 1. Linearized perturbations, Phys. Rev. D 49 (1994) 883 [gr-qc/9307009] [InSPIRE]. 
[30] C. Gundlach, R.H. Price and J. Pullin, Late time behavior of stellar collapse and explosions: 2. Nonlinear evolution, Phys. Rev. D 49 (1994) 890 [gr-qc/9307010] [InSPIRE].

[31] E.W. Leaver, Spectral decomposition of the perturbation response of the Schwarzschild geometry, Phys. Rev. D 34 (1986) 384 [InSPIRE].

[32] M. Dafermos and I. Rodnianski, A proof of Price's law for the collapse of a selfgravitating scalar field, Invent. Math. 162 (2005) 381 [gr-qc/0309115] [INSPIRE].

[33] Y. Angelopoulos, S. Aretakis and D. Gajic, Logarithmic corrections in the asymptotic expansion for the radiation field along null infinity, J. Hyperbol. Diff. Equat. 16 (2019) 1 [arXiv: 1712.09977] [INSPIRE].

[34] L.M.A. Kehrberger, The case against smooth null infinity II: a logarithmically modified price's law, arXiv:2105.08084 [INSPIRE].

[35] L.M.A. Kehrberger, The case against smooth null infinity III: early-time asymptotics for higher $\ell$-modes of linear waves on a Schwarzschild background, arXiv:2106.00035 [INSPIRE]. 